\title{
COVID-19 vaccine hesitancy and missed vaccine roll- out targets: The case for using general practitioners
}

\author{
D J McQuoid-Mason, BComm, LLB, LLM, PhD \\ Centre for Socio-Legal Studies, University of KwaZulu-Natal, Durban, South Africa
}

Corresponding author: D J McQuoid-Mason (mcquoidm@ukzn.ac.za)

\begin{abstract}
The South African (SA) government's roll-out of the COVID-19 vaccine is behind its target, largely owing to concerns about the sideeffects and the effectiveness of the vaccines, and because they have been developed over a very short time frame. Another factor is a lack of trust in government policies regarding COVID-19 and its running of public health. One survey has indicated that for persons seeking a vaccination, the preferred vaccine site would be general practitioners (GPs). GPs have been used in Australia, the UK and elsewhere. In Australia, with a scattered rural population, $5600 \mathrm{GPs}$ have been vaccinating over one million patients weekly. Calls have been made by the South African Medical Association, among others, for GPs to be allowed to assist with the government's roll-out programme. If $\sim 8000$ GPs in SA participated in a properly administered roll-out programme, and each GP were to vaccinate only 10 people a day, this would yield 400000 vaccinations a week or $\sim 1.6$ million a month. The GPs could invite their patients and others to visit their room for a COVID-19 vaccination, as they do with the annual influenza vaccine.
\end{abstract}

S Afr Med J. Published online 27 January 2022. https://doi.org/10.7196/SAMJ.2022.v112i3.16379

The South African (SA) government is falling behind with its COVID-19 vaccination roll-out targets, despite using several innovative strategies to achieve them. The glaring gap in these measures, however, is the failure by the Department of Health (DoH) to utilise one of the country's most trusted and effective health resources, that are used in several other countries - general practitioners (GPs). This failure has persisted despite the South African Medical Association (SAMA) and others calling on government to allow GPs to assist with the vaccine roll-out and stating that the roll-out programme should be less centrally controlled by government. ${ }^{[1,2]}$

In considering whether it would be useful to involve GPs, it is necessary to examine: (i) the reasons for COVID-19 vaccination hesitancy; (ii) the level of public confidence in the DoH and government policies; (iii) public confidence in the services provided by GPs; and (iv) the steps the DoH can take to ensure that GPs provide a proper service.

\section{Reasons for vaccination hesitancy}

Many reasons have been advanced for COVID-19 vaccine hesitancy in other countries. In the USA, the most widely reported concern was that the side-effects of COVID-19 vaccines would be worse than those of the virus itself. Many people, including healthcare workers, were worried about how effective the vaccines were. There was also a concern that the vaccines had been developed too quickly and had been approved too rapidly by the regulation authorities, and that there was a lack of transparency on the vaccines in general and allergic reactions to them in particular. ${ }^{[3]}$

A comprehensive survey of vaccine hesitancy in SA yielded similar results to those in the USA. The University of Johannesburg (UJ) and the Human Sciences Research Council (HSRC) conducted an analysis of vaccine hesitancy in SA. ${ }^{[4]}$ They found that the main reasons for COVID-19 vaccine hesitancy in SA were also concerns about the side-effects, the overall effectiveness of the vaccine, and that the vaccines are 'moving through clinical trials too fast'. Similar reasons were found in other surveys. ${ }^{[5]}$
An important finding in the UJ-HSRC survey was that $50 \%$ of respondents indicated that GPs would be their preferred vaccination site, while $33 \%$ of respondents preferred pharmacies. The remaining $17 \%$ 'chose hospitals, clinics, community centres and other types of settings as their preferred vaccination sites. ${ }^{[4]}$

\section{Level of public confidence in DoH and government COVID-19 initiatives}

International and local surveys have shown that the public has little confidence in the ability of the state to provide safe and effective health services. The same applies to the SA government's policies regarding COVID-19, including its vaccination policy. ${ }^{[2,4]}$ Many people believe that they are being vaccinated during a large clinical trial being conducted by the DoH's partners to determine how effective the vaccines are. ${ }^{[6]}$ People also believe that children should not have to be vaccinated because they do not suffer serious illness or die from COVID-19. ${ }^{[7]}$

\section{Level of public confidence in the} services provided by GPs

The results of the UJ-HSRC survey indicate that in SA, GPs are the most trusted sector in the health services for the administration of the COVID-19 vaccine. ${ }^{[7]}$ People tend to trust their GPs more than any other providers of health services. If the DoH sincerely wishes to increase the uptake of vaccinations by the public, it should allow GPs to administer the vaccine like they do with influenza vaccinations. GPs could then invite their patients and others to visit their rooms to be vaccinated. In 2016 there were 8721 GPs in solo and group private practices in $\mathrm{SA},{ }^{[8]}$ and there are probably considerably more in 2022. They could play a valuable role in persuading their patients and others to take the vaccine and educating them about its importance. They could significantly boost the numbers of people who take the vaccine, as has happened in Australia. ${ }^{[9]}$

In Australia, 5600 GPs have been involved in administering COVID-19 vaccines in rural, regional and metropolitan areas 
throughout the country. ${ }^{[9]}$ Like SA, Australia has populations scattered across large rural areas. The Australian GPs involved in the COVID-19 roll-out programme have administered a weekly average of over one million vaccines..$^{[9]}$

\section{Steps the DoH can take to ensure that GPs are providing a proper service}

The UK began using GPs to roll out the COVID-19 booster vaccination programme in December 2021. The GPs are required to have sufficient space to seat patients who have received the vaccine and have to wait 15 minutes to monitor any adverse side-effects, and proper cold-storage facilities. There were initial delivery problems with getting stocks of vaccines to GP surgeries on time. ${ }^{[10]}$ SA could learn from the $\mathrm{UK}^{[10]}$ and Australian ${ }^{[9]}$ experiences, and consider implementing a similar programme for accredited private GPs.

If managed properly, over 8000 GPs in private practice in $\mathrm{SA}^{[8]}$ should exceed the Australian weekly administration of over one million doses by 5600 GPs. If 8000 GPs administered only 10 vaccinations a day, they could administer 400000 doses a week, or $\sim 1.6$ million doses a month. For this to happen, SAMA has pointed out that the electronic vaccination data system would have to be upgraded 'to allow doctors to register more easily', and doctors should be allowed to buy vaccines in batches of 10 - 15 doses rather than in bulk, which is currently required. ${ }^{[1]}$

If GPs in SA become involved in the COVID-19 vaccination roll-out programme, patient confidence in the COVID-19 vaccine is likely to be boosted by knowing that their GPs already offer other safe vaccination services to patients, such as annual influenza vaccinations. ${ }^{[2]}$
Declaration. None.

Acknowledgements. None.

Author contributions. Sole author.

Funding. National Research Foundation.

Conflicts of interest. None.

1. Staff reporter. Allow GPs to give Covid vaccines: South African Medical Association. Businesstech, 4 January 2022. https://businesstech.co.za/news/trending/548472/allow-gps-to-give-covid-vaccinesuth-african-medical-association/ (accessed 8 January 2022)

Geffen N, Low M. How to vaccinate millions as quickly as possible. Daily Maverick, 20 May 2021. https://www.dailymaverick.co.za/article/2021-05-14-how-to-vaccinate-millions-as-quickly-aspossible/ (accessed 2 January 2022).

3. Wang Y, Liu Y. Multilevel determinants of COVID-19 vaccination hesitancy in the United States: A rapid systematic review. Prev Med Rep 2021;25:101673. https://doi.org/10.1016/j. pmedr.2021.101673

4. Cooper S. Covid-19 vaccine hesitancy in South Africa. 20 April 2021. https://sacoronavirus.co.za/ wp-content/uploads/2021/04/Report_Covid-19-vaccine-hesitancy_SA-studies_1April2021.pdf (accessed 2 January 2021).

5. Cooper S, van Rooyen H, Wiysonge CS. COVID-19 vaccine hesitancy in South Africa: How can we maximize uptake of COVID-19 vaccines? Exp Rev Vaccines 2021;20(8):921-933. https://doi.org/10. 1080/14760584.2021.1949291

6. Reddy DL, Dangor Z, Lala N, Johnstone J, Maswabi L. COVID-19 mass vaccination campaign for healthcare workers in a low-resource setting: A clinician-driven initiative. S Afr Med 2021;111(7):610-614. https://doi.org/10.7196/SAMJ.2021.v11117.15712

7. Venter Z. High Court bid to halt vaccine roll-out to children. Pretoria News, 3 November 2021.

8. Moosa S, Luiz J, Carmichael T, Peersman W, Derese A. Insights of private general practitioners in group practice on the introduction of National Health Insurance in South Africa. Afr J Prim Health Care Fam Med 2016;8(1):a1025. https://doi.org/10.4102/phcfm.v8i1.1025

9. Kidd M, de Toka L. The 'significant' role GPs have in exiting pandemic. NewsGP, Australia, 30 November 2021. https://www1.racgp.org.au/newsgp/gp-opinion/the-significant-role-gps-have-inexiting-pandemic (accessed 1 January 2022).

10. Mahase E. Covid-19: Logistical problems frustrate GPs ready to deliver vaccine in England. BMJ 2020;371:m4849. https://doi.org/10.1136/bmj.m4849

Accepted 24 January 2022 